\section{Gesellige Methode}

\author{
FOKUSGRUPPEN Viele Themen lassen sich kontrovers \\ diskutieren. Um diese Meinungsvielfalt zu erfassen, bieten \\ sich Fokusgruppen an - eine wissenschaftliche Erhebungs- \\ methode mit geselligem Charakter.
}

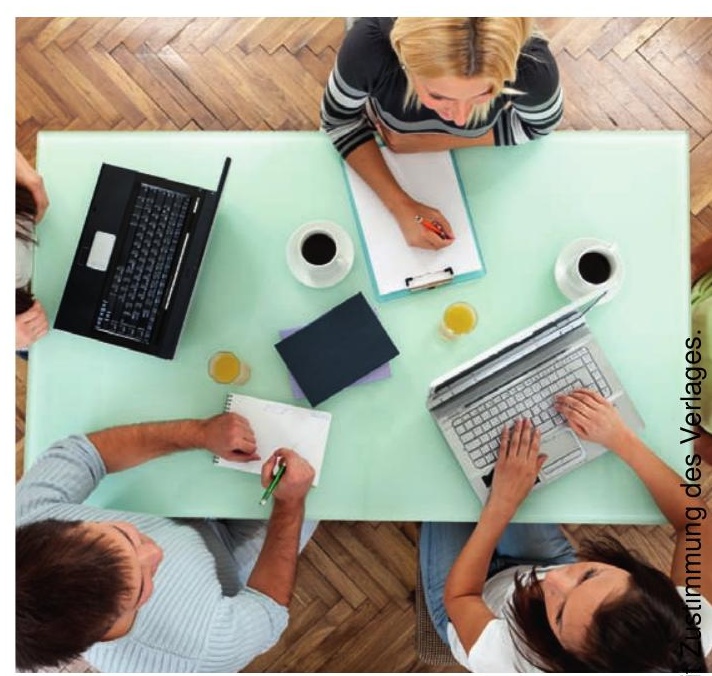

M iteinander diskutieren, sich austauschen, gemeinsam überlegen - das klingt fast danach, als würden gute Freunde bei einer Tasse Tee oder Kaffee zusammensitzen. Getränke und Knabbereien können auch in einer Fokusgruppe auf dem Tisch stehen. Gegenüber einem Treffen unter Freunden erfordert diese Erhebungsmethode aber, die Teilnehmer gezielt auszuwählen und die Diskussionsinhalte vorzubereiten und auszuwerten (॰ „Ablaufschema für Fokusgruppen“).

Eine Studie besteht meist aus mehreren Fokusgruppen, in denen die Teilnehmer über ein vorgegebenes Thema diskutieren [1]. Vorab erhalten sie gezielten Input, der sie ins Thema einführt und die Diskussion entfacht [2].

Der amerikanische Soziologe Robert Merton gilt als einer der Ersten, der Fokusgruppen zu Forschungszwecken einsetzte. Er untersuchte in den 1940er Jahren, wie Zuschauer auf Kriegspropaganda reagierten. In den darauffolgenden Jahrzehnten verbreitete sich diese Methode vor allem in der US-amerikanischen Marktforschung. Mittlerweile haben sich Fokusgruppen auch in der internationalen Sozial-, Gesundheits- und Medienforschung etabliert und begegnen uns in vielen therapeutischen Studien. Die deutschsprachige Forschungsliteratur bevorzugt allerdings den Begriff „Gruppendiskussion“, den man nicht eindeutig von der Fokusgruppe abgrenzen kann [2, 3].

Vielseitig einsetzbar > Bei Fokusgruppen geht es um zwei Dinge: Man konzentriert sich auf ein Thema und nutzt die Gruppeninteraktion, um Daten zu erheben [2]. Dabei ermittelt man, welche Gefühle und Gedanken
Menschen zu einem bestimmten Thema entwickeln oder welche Normen und Erfahrungen in einer Gruppe existieren [4, 5]. Ein ergotherapeutisches Forschungsteam um Gunilla Liedberg untersuchte beispielsweise in Schweden, wie Ergotherapeuten das Gender-Konzept wahrnehmen [6].

Fokusgruppen eignen sich besonders für Problemstellungen, zu denen bislang wenig gesichertes Wissen vorliegt. Mit ihrer Hilfe kann man das Meinungsspektrum der Teilnehmer erfassen und wichtige Aspekte identifizieren, die mit dem ausgewählten Thema in Verbindung stehen. Daher stellen sie oft den Ausgangspunkt für weiterführende Forschungen dar. Sie bieten sich aber auch in der Abschlussphase größerer Projekte an, um Studienergebnisse zu interpretieren oder Feedback von den Teilnehmern einzuholen [5, 7].

Mit der gewählten Problemstellung entscheiden Forscher darüber, wer an einer Fokusgruppe teilnehmen soll. So untersuchte Mirtha Whaley an der Universität Florida, USA, wie Ergotherapiestudenten die Arbeit mit älteren Menschen erleben. Wer könnte darüber besser Auskunft geben als diese selbst? Deshalb organisierte sie fünf Fokusgruppen mit 37 Ergotherapiestudenten, die über unterschiedliche Ausbildungsgrade verfügten [8].

Interaktion ermöglichen > Zu einer Problemstellung finden normalerweise mehrere Fokusgruppen statt, die sich aus unterschiedlichen Teilnehmern zusammensetzen. In der Sozialforschung findet man aber auch die Variante, dass dieselbe Gruppe mehrere Erhebungsrunden durchläuft [2].
Damit die Fokusgruppen ihr Ziel erreichen, müssen die Gruppenmitglieder miteinander in einen lebendigen Austausch treten. Sie unterhalten sich angeregt, lachen, beschreiben eigene Erfahrungen oder widersprechen einander [9]. Häufig bestehen die Diskussionsrunden aus Teilnehmern, die über einen ähnlichen Hintergrund verfügen und beispielsweise einen vergleichbaren Beruf ausüben. Solche homogenen Gruppen erleichtern es, miteinander ins Gespräch zu kommen [2]. Im Idealfall setzen sie sich aus sechs bis zehn Teilnehmern zusammen, damit jeder ausreichend Redezeit erhält [1]. Die Diskussion sollte in einem neutralen Raum stattfinden und maximal zwei Stunden dauern, sodass die Teilnehmer ihr aufmerksam folgen können [5]. Neben den persönlichen Treffen setzen sich zunehmend auch Online-Verfahren durch [10].

Damit die Beteiligten besser ins Thema finden, erhalten sie vor der Diskussion einen Input. Dieser kann aus Videos, Texten oder Bildern bestehen und soll das Bedürfnis stimulieren, sich mit anderen auszutauschen [2]. Der anschließende Gruppenprozess ermutigt die Teilnehmer dazu, das Thema aus unterschiedlichen Blickwinkeln zu betrachten und kontrovers zu diskutieren.

Auf den Moderator kommt es an > Der Gruppenprozess bringt nicht nur Vorteile mit sich, sondern auch Risiken. So kann eine Gruppensituation einzelne Teilnehmer hemmen, ihre Meinung frei zu äußern. Unter Umständen bilden sich informelle Meinungsführer heraus, oder es entstehen Mechanismen der sozialen Kontrolle. Der Erfolg einer 


\section{physiowissenschaft}

\section{Ablaufschema für Fokusgruppen [2]}

Phase 1:

$>$ Problem definieren und Forschungsfrage

formulieren

$>$ Gruppe bestimmen

$>$ Moderatoren auswählen und ausbilden

$>$ Leitfaden und Input erstellen

$>$ Pretest durchführen

$>$ Teilnehmer rekrutieren
Phase 2:

$>$ Diskussion durchführen

Phase 3:

$>$ Daten analysieren und interpretieren

$>$ Ergebnisse zusammenführen und ggf. präsentieren
Fokusgruppe hängt daher ganz entscheidend vom Moderator ab. Er muss nicht vom Fach sein, sollte sich aber mit Moderationstechniken auskennen und dafür sorgen, dass ein konstruktives Gesprächsklima herrscht und sich alle Gruppenmitglieder in die Diskussion einbringen können. Dann kann die Fokusgruppe ihre große Stärke offenbaren: die Teilnehmer provozieren, spontan auf die Beiträge anderer zu reagieren und Meinungen authentisch zu äußern [3, 5].

Statt zu kontrollieren, sollte der Moderator die Diskussion anregen und bedarfsweise wieder zum Thema zurückführen. Als Orientierungshilfe erhält er dazu einen Leitfaden, den die Forscher im Vorfeld entwickeln und in einem sogenannten Pretest überprüfen

\section{(॰ „Ablaufschema für Fokusgruppen“).}

Die benötigten Daten entstehen, während die Teilnehmer sich austauschen. Videoaufnahmen oder Audiotapes dienen dazu, die Diskussion bis in ihre Einzelheiten festzuhalten [2]. Als zusätzliche Strukturierungshilfe bieten sich Mapping-Techniken an, um die Beiträge zu bündeln und zu visualisieren [11].

Anstöße für Theorie und Praxis > Die ausgewerteten Daten können einen Einblick in individuelle und kollektive Meinungsbilder eröffnen. Durchläuft die Fokusgruppe mehrere Erhebungsrunden, dienen sie als Grundlage für den neuen Leitfaden.

Fokusgruppen liefern wichtige Erkenntnisse darüber, wie Menschen ihre Wirklichkeit konstruieren und welche Normen und Erfahrungen in einer sozialen Gruppe existieren [3, 5]. Ihre Ergebnisse können weitere Forschungen anregen und in Ausbildungs- oder Behandlungs- programme einfließen [8, 9, 12]. Mitunter tragen sie auch dazu bei, neue Assessments oder Modelle zu entwickeln. Während der Entstehung seines sogenannten KAWA-Modells initiierte Professor Michael Iwama beispielsweise mehrere Fokusgruppen mit japanischen Ergotherapeuten, um deren Verständnis von Ergotherapie herauszufinden [13].

Da Fokusgruppen vor allem subjektive Meinungen und Erfahrungen ermitteln, besitzen sie eine eher geringe Beweiskraft. Als alleinige Methode sind sie daher weniger geeignet, um die Wirkungen von Interventionen zu evaluieren oder miteinander zu vergleichen. Gegenüber anderen Erhebungsinstrumenten haben sie aber einen großen Vorteil, den man aus Diskussionen im Freundeskreis oder Team kennen dürfte: Der direkte Austausch regt an oder sogar auf. Er lässt Meinungen und Ideen zutage treten, die einem bislang nicht bewusst waren. Im Dialog kann man sie entdecken und weiterentwickeln.

Florence Kranz

\section{$\Rightarrow$ Literatur: www.thieme-connect.de/ ejournals/physiopraxis}

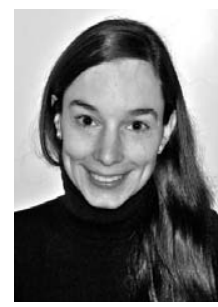

Florence Kranz, Ergotherapeutin BcOT und cand M.A. Gesundheitsmanagement, hat mehrere Bachelorarbeiten betreut, in denen auch Fokusgruppen zum Einsatz kamen. 